\title{
Influence of laser irradiation on the activity of plant pharmaceuticals with the assessment by the bacteria growth dynamics
}

\author{
Lubov A. Kokorina and Anna V. Neupokoeva* \\ Irkutsk State Medical University, 1 Krasnogo Vosstaniya Str., Irkutsk, 664009 Russia \\ * e-mail: annett2005@inbox.ru
}

\begin{abstract}
The article describes experimental investigation of influence of laser irradiation on the drug activity of umbellate wintergreen and roundleaved wintergreen in the form of decoction and extract. The drug activity is estimated by counting the number of colonies grown on the nutrient medium with the addition of the studied plant preparation. It is shown that laser irradiation of pharmaceuticals significantly changes their activity, and the best results are obtained by irradiation of the umbellate wintergreen decoction. (C) 2019 Journal of Biomedical Photonics \& Engineering.
\end{abstract}

Keywords: laser; drug activation.

Paper \#3315 received 10 Feb 2019; revised manuscript received 17 Mar 2019; accepted for publication 3 Apr 2019; published online 13 May 2019. doi: 10.18287/JBPE19.05.020302.

\section{Introduction}

Modern trends of medical and biological laser radiation application can be divided into two main groups. The first group uses unique properties of laser radiation as a research tool: spectral analysis, laser microscopy, holography, etc. The second group uses laser radiation for influence on biological objects: in therapy, surgery and diagnosis.

At present, many researchers have shown in practice that complex using of laser radiation and standard drugs for the treatment of various human diseases is more effective than their separate using [1-7]. This approach is often due to the fact that modern antibiotics are not always effective, since most bacteria have learned to defend themselves. So some strains of Staphylococcus aureus acquired resistance to a wide range of antibiotics, in particular to penicillins (methicillin, dicloxacillin, nafcillin, oxacillin, etc.) and cephalosporins. This leads to various complications after staphylococcal infection and causes high mortality. Therefore, search for new drugs or modification of existing ones under influence of laser radiation is an important direction.

On the other hand, most drugs are used in the form of liquids: solutions, emulsions, extracts, etc. When large organic molecules (primarily proteins and polypeptides) dissolve in water, a solution like colloidal is formed. At the micro level, this means that protein macromolecules do not break down into simpler components but rather acquire a hydrate shell transferring part of the water molecules into a bound state. Thus, dissolved substances form clusters (associates) by combining molecules due to electrostatic interaction forces $[8,9]$. A cluster can consist of several tens of molecules, which leads to decreasing their mobility and reactivity, because active centers can be overlapped by near-located molecules. Therefore, destruction of clusters into components would increase biochemical activity of molecules, and hence activity of the solution as a whole.

As a rule, the molecules in the cluster are bound by hydrogen or hydrophobic interactions, so such formations are unstable and must be destroyed by physical factors. Thus, it is known $[10,11]$ that under action of laser radiation clusters are able to decay into smaller formations, since coherent monochromatic laser radiation causes intensive movement of charged areas of macromolecules, as a result of which molecules break out of clusters, creating a homogeneous mixture with fewer inhomogeneities.

The aim of our work is assessing change in the plant drugs activity of umbellate wintergreen (Chimaphila umbellate (L.) W. Barton) and round-leaved wintergreen (Pyrola Rotundifolia) to Staphylococcus aureus under influence of laser radiation. 


\section{Experiment}

\subsection{Materials and methods}

For experimental studies, we used reference strain Staphylococcus aureus - gram-positive, regular shape globular cells in the smear are arranged in clusters in the form of "grapes". They are included in the group of conditionally pathogenic microorganisms, which are sensitive to ampicillin. Temperature optimum for bacterial growth is $25-35{ }^{\circ} \mathrm{C}$, optimum $\mathrm{pH}$ is $7.0-7.5$. Meat-peptone agar (MPA) was used to cultivate bacteria.

Impact on plant drugs before its adding to nutrient medium was carried out in the "light boiler" mode. The duration of exposure was 20 and 60 seconds. The radiation power was about $100 \mathrm{~mW}$, the wavelength was $532 \mathrm{~nm}$, and total irradiation energy was about $5 \mathrm{~J} / \mathrm{g}$.

The choice of plants as a drugs source was due to the fact that both the umbellate wintergreen and roundleaved wintergreen are widely used as antiseptics, antiinflammatory and antimicrobial drugs. Their composition is well known, in particular, both plants contain flavonoids, tannins, triterpenoids. All these components can provide antimicrobial activity [12].

There were two types of umbellate wintergreen preparations: herbal decoction and alcohol tincture (extract) in experiments. The concentration of the active substance in the decoction was $0.025 \%$, and in the extract $-0.06 \%$. Also in experiments, two types of round-leaved wintergreen preparations were used: herbal decoction and alcohol tincture (extract). The concentration of the active substance in the decoction and in the extract was the same $-0.01 \%$. These drugs were exposed by laser radiation for 20 and 60 seconds. Control (C) was a sterile physiological solution, positive control $(\mathrm{C}+)$ was ampicillin solution (penicillin semisynthetic) - broad-spectrum antibiotic at a concentration of $1 \mu \mathrm{g} / \mathrm{ml}$.

In the experiments by scheme 1 we studied the colonies grow dynamics on MPA. $1 \mathrm{ml}$ of bacterial suspension and $1 \mathrm{ml}$ of the test solution were added to the flask with the nutrient medium. The flask's contents were mixed and poured into Petri dishes, which then were placed in a thermostat $\left(37-38{ }^{\circ} \mathrm{C}\right)$. Colonies number counting was made every 12 hours.

In the experiments by scheme 2, we estimated influence of laser radiation on the drugs antibacterial activity by measuring of the growth inhibition zone [13]. $1 \mathrm{ml}$ suspension of microorganisms was added to the flasks with $100 \mathrm{ml} \mathrm{MPA}$, and then the resulting mixture was poured into Petri dishes. After agar gelation three holes of the same diameter were made in the MPA medium. Non-irradiated and irradiated drugs were added in the holes and then Petri dishes were put in thermostat under $37^{\circ} \mathrm{C}$. After 12 hours we measured the diameter of growth inhibition zones with accuracy within $1 \mathrm{~mm}$.

\subsection{Results and discussion}

During the experiments the colonies growth dynamics on the agar medium (MPA) was recorded by measuring the total number of grown colonies every 12 hours. Further, the obtained data were averaged over three series of experiments, the error did not exceed $11 \%$ (Fig. 1).

Data comparison (Fig. 1) shows that the irradiated umbellate wintergreen decoction inhibits the St. aureus colonies growth. The most pronounced effect was observed under the action of the drug irradiated for 60 seconds (the number of colonies is $12-14 \%$ of the control). Whereas, in the experiment with non-irradiated decoction colonies number was more than control ( $103 \%$ for 12 hours after the cultivation start).

Under the action of the not irradiated extract the grown colonies number was $64 \%$ of the control by the end of the observations. It represents a weak bactericidal effect compared to the irradiated drug for that the number of bacterial colonies was $33 \%$ and $42 \%$ of the control for time exposure 20 seconds and 60 seconds respectively. Thus, irradiated umbellate wintergreen extract inhibits the bacteria growth stronger than not irradiated, and the laser exposure duration doesn't play a significant role, because the difference is comparable to the measurement error. Ampicillin inhibits the growth of St. aureus completely.

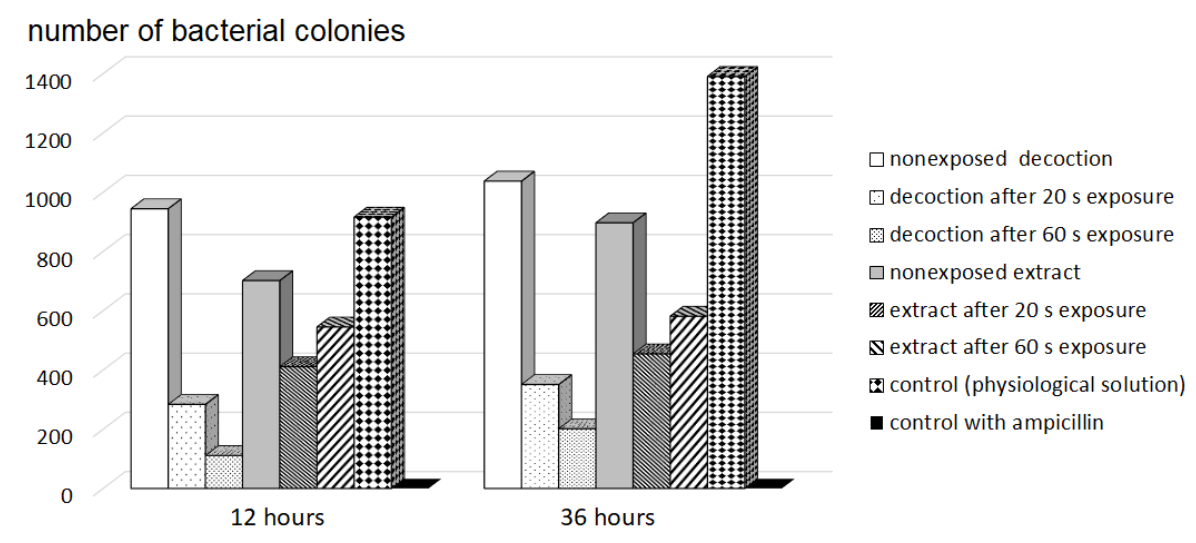

Fig. 1 Colonies growth dynamics on agar medium with the addition of decoction and extract of umbellate wintergreen. 
Photos of grown colonies on MPA in the analyzed experiments are presented in Fig. 2. Experiments was carried out by the diffusion in agar method. Drugs antimicrobial activity was recorded by size measurement of Staphylococcus growth inhibition zone [13], which was formed after 12 hours of cultivation.

Growth inhibition zones were not found in experiments with non-irradiated umbellate wintergreen decoction, as well as in the control version. Laser irradiation of the decoction led to the bactericidal activity appearance: the growth inhibition zones were 12 and $13 \mathrm{~mm}$ and bactericidal activity did not significant depend on exposure time. Ampicillin $(\mathrm{K}+)$ actively suppresses the Staphylococcus aureus growth, the suppression zone size was from 23 to $30 \mathrm{~mm}$. Ampicillin bactericidal activity was not changed under laser exposure for 20 and 60 seconds.
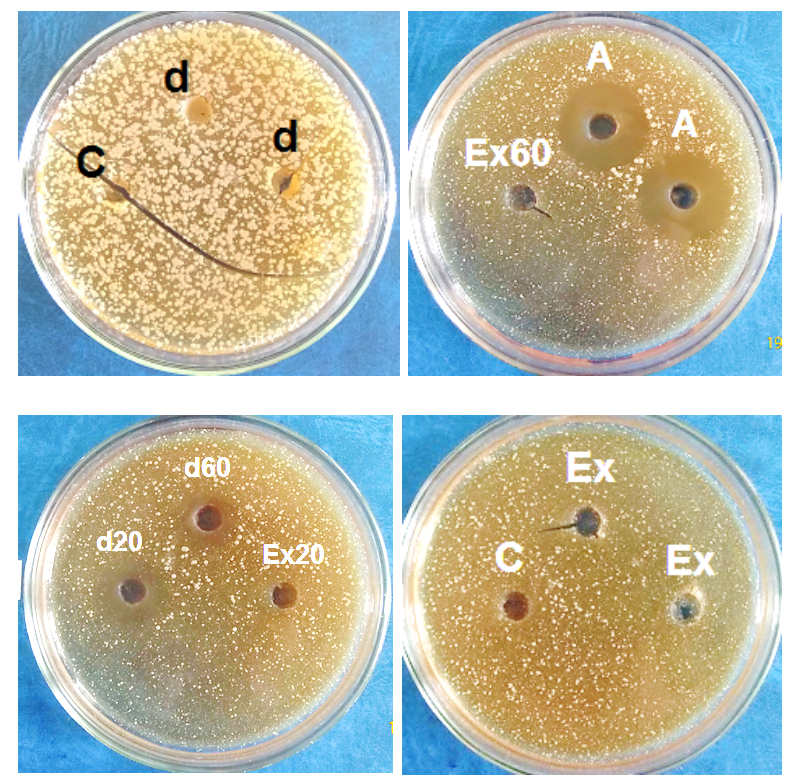

Fig. 2 Growth inhibition zone of the experiments with umbellate wintergreen drugs: $\mathrm{C}$ - control (physiological solution), d - non exposed wintergreen decoction, d20 - wintergreen decoction after 20 second exposure, d60 - wintergreen decoction after 60 second exposure, Ex - non exposed wintergreen extract, Ex20 - wintergreen extract after 20 second exposure, Ex60 - wintergreen extract after 60 second exposure, A - control with ampicillin.

Experimental data for round-leaved wintergreen are shown in Fig. 3, the error does not exceed $26 \%$. According to the diagram (Fig. 3) on the first day the bactericidal effect is expressed in the non-irradiated and irradiated round-leaved wintergreen decoction, as well as in the non-irradiated round-leaved wintergreen extract: the number of colonies is $60-80 \%$ of the control. At the same time, irradiated extract stimulate the St. aureus growth (133\% compared to control). After 48 hours from the start of observation roundleaved wintergreen decoction, both irradiated and nonirradiated, retain bactericidal activity. And, preparations based on the extract do not have any stimulating or bactericidal effect, i.e. do not cause significant changes in the bacteria growth compared to the control.

Ampicillin completely inhibits the growth of bacteria.

Bactericidal activity registration by diffusion in agar method was carried out 12 hours after the cultivation start. The experiment showed that non-irradiated and irradiated preparations of round-leaved wintergreen do not lead to the appearance of growth inhibition zones.

\section{Conclusions}

Bactericidal activity of plant preparations on the base of umbellate wintergreen and round-leaved wintergreen in two dosage forms (decoction and alcohol extract), irradiated with low-intensity laser radiation was investigated. The minimum colonies growth was observed under the action of 20 and 60 seconds irradiated umbellate wintergreen preparations (decoction and alcohol extract). It was also found that the non-irradiated umbellate wintergreen decoction did not lead to the suppression of St. aureus growth, but laser irradiation of the drug causes bactericidal activity appearance, which is significantly different from the control. Non-irradiated extract leads to weak inhibition of St. aureus growth, but the irradiated drug bactericidal activity increases almost 2 times in comparing with nonirradiated, regardless of the laser exposure time.

Bactericidal action of umbellate wintergreen decoction was not revealed by the diffusion in agar method, while the irradiated drug caused the growth inhibition zones appearance. Under the diffusion in the culture medium of non-irradiated umbellate wintergreen extract and irradiated preparations bactericidal action is not revealed.

A moderate bactericidal effect was found under applying a round-leaved wintergreen decoction, but statistically significant differences caused by laser irradiation of the drug compared with the control was not detected.

It is necessary to note that the bactericidal activity of drugs is due to the whole complex of components of the plant raw materials. Without special studies it is impossible to separate a single component that suppresses the bacteria growth. According to the literature data [12], bactericidal activity can be provided by flavonoids and triterpenoids, and the inhibition mechanisms of bacterial growth can be different: direct membranes destruction, binding to protein complexes, which violates the specific function of the protein, violation of enzyme - substrate interaction by binding to one of them. In all these cases, the drug activity is affected by the number of molecules that can interact with the membrane or proteins, as well as their aqueous environment. The combination of several molecules to a cluster surrounded by a hydrate shell reduces their activity.

Decoction-based preparations contain about $80 \%$ water and do not contain ethanol. Preparations based on the extract contain only $56 \%$ water and $38 \%$ alcohol (ethanol). Therefore, in alcohol extracts, the 
antibacterial activity of molecules can be reduced by interacting with ethanol, which not only changes the oxidative activity of organic molecules, but also transforms some water into a bound state.

A significant difference in the bactericidal action of the umbellate wintergreen and round-leaved wintergreen drugs due to different chemical composition and ratio of components. However, the phenomenon of bacterial growth stimulation by irradiated round-leaved wintergreen extract, despite the fact that the non-irradiated extract, on the contrary, has a bactericidal effect, requires further research.

\section{Disclosures}

All authors declare that there is no conflict of interests in this paper.

\section{References}

1. A. V. Nikitin, M. F. Gorbatykh, E. F. Evstratova, and S. A. Fisunova, "Changes in pro-inflammatory cytokines and oxidative status in patients with psoriatic arthritis during treatment with a combination of arthrofoon with lowintensity laser radiation," Bulletin of new medical technologies 19(2), 260-263 (2012) [In Russian].

2. Y. N. Chernykh, V. A. Valuyev, "Evaluation of the effectiveness of low-intensity laser radiation in the complex treatment of patients with chronic obstructive pulmonary disease," Bulletin of new medical technologies 18(2), 323-325 (2011) [In Russian].

3. A. V. Lebedeva, D. A. Malyukov, and A. V. Nikitin, "Low-intensity laser radiation and immunofuran in the complex therapy of chronic obstructive pulmonary disease," Bulletin of new medical technologies17(2), 214-216 (2010) [In Russian].

4. G. V. Maksimov, A. V. Nagovitsyn, "The role of low molecular weight proteins in the implementation of the action of laser radiation and an alternating magnetic field on the blood," Bulletin of Moscow University. Series 16: Biology 3, 8-12 (2009) [In Russian].

5. G. A. Zalesskaya, E.G. Sambor, "Interaction of low-intensity laser radiation with blood and its components," Journal of Applied Spectroscopy 72(2), 230-235 (2005).

6. V. F. Kirichuk, O. V. Khlebina, "The nature of changes in endogenous intoxication, lipid peroxidation in patients with inflammatory processes of the uterus appendages in combination with standard treatment with helium-neon laser radiation," Basic research 11, 69-73 (2010) [In Russian].

7. A. P. Manzhos, Optimization of the use of low-intensity infrared laser radiation in the treatment of bronchial obstruction with a stable course of chronic obstructive pulmonary disease, PhD thesis, Moscow (2007) [In Russian].

8. A. V. Finkelshtein, O.B. Ptitsyn, Protein Physics, Knizhyi dom "Universitet", Moscow, Russia (2002) [in Russian].

9. V. D. Lakhno, Clusters in Physics, Chemistry, Biology, NITs "Regular and chaotic dynamics", Izhevsk, Russia (2001) [in Russian].

10. A. N. Malov, A. A. Vaichas, Laser Control of Biomineral Formation. Physical Principles and Prospects of Developing New Medical Nanotechnology, Palmarium Academic Publishing, Saarbrucken, Deutschland (2016) (ISBN 978-3-659-60476-8) [in Russian].

11. A. N. Malov, A. Y. Seteikin, A. V. Neupokoeva, E. S. Musatova, I. E. Golub, L. V. Sorokina, V. S. Fetschenko, and A. A. Vaichas, "The laser radiation action on the biological objects," Optik 124(23), 6034-6041 (2013).

12. M. M. Cowan, "Plant Products as Antimicrobial Agents," Clinical Microbiology Reviews 12(4), 564-582 (1999).

13. Determination of antimicrobial activity of antibiotics using the method of diffusion into agar. State Quality Standard of Medical Agent. General Pharmacopeia Article, Ministry of Health of Russian Federation (2010) [in Russian]. 